“C 2010 IEEE. Personal use of this material is permitted. Permission from IEEE must be obtained for all other uses, in any current or future media, including reprinting/republishing this material for advertising or promotional purposes, creating new collective works, for resale or redistribution to servers or lists, or reuse of any copyrighted component of this work in other works.” 


\title{
Laboratory Demonstration for Model Predictive Multivariable Control with a Coupled Drive System
}

\author{
Steven W. Su†, Hung T. Nguyen $\dagger$, and Q. P. Ha† \\ $\dagger$ Faculty of Engineering and Information Technology \\ University of Technology, Sydney \\ Broadway NSW 2007 Australia \\ (Steven.Su, Hung.Nguyen, quangha)@eng.uts.edu.au
}

\begin{abstract}
Teaching multivariable control usually involves a certain level of mathematical sophistication and hence requires some labaratorial exemplification of the material given in formal lectures. This paper reports on a hands-on approach to multivariable control education via the implementation of a model predictive controller on a two-input, two output coupled drive apparatus. This scaled-down system represents many industrial processes while provides an excellent set-up for demonstrating the cross-coupled effects in multi-input multi-output systems. Here, a model predictive controller (MPC) is developed and implemented on the basis of a constrained optimization problem to show control performance via the belt tension and velocity outputs, demonstrate the decoupling capability, and also illustrate such issues as control input saturation, the selection of operating point, reference inputs, and system robustness to external disturbance and varying parameters. The implementation is based on Labview and MATLAB Model Predictive Control Toolbox.
\end{abstract}

Index Terms-Multivariable control, Optimization, Nonlinearity, Model predictive Control, Coupled drives.

\section{INTRODUCTION}

Multi-input multi-output (MIMO) systems remain an important topic in control engineering education. To complement the theoretical aspects and rigorous treatments in MIMO control, it is essential to provide students with some laboratorial demonstration and practical work. This paper reports on the development of a model predictive controller for a two-input twooutput coupled drive apparatus for that purpose [1]. Regulation of both the belt tension and speed of a coupled drive system is commonly encountered in many continuous automation processes in industries such as textile manufacturing, paper making, strip metal and wire manufacturing, plastic films, food packaging and production line. Coupled drive systems exhibit intercoupling effects that are typical in multivariable control whereby advanced techniques have been applied to deal with a number of outputs at the same time, e.g., fuelinjection quantity, pressure, and timing waste cut and energy economy (http://machinedesign.com/article/pumping-new-lifeinto-mobile-machines-0605). In our laboratory a coupled drive model (www.control-systems-principles.co.uk) is employed for the laboratory teaching of control engineering subjects. As pointed in [1], teaching of multivariable systems is a dedicated effort requiring a balance of complex, abstract elaboration and hands-on experience as the topics may in part incur a perceived jump in mathematical sophistication required of students and the uneasy fit with prior learned classical control material [1].
In the control development for coupled drive systems, the commonly-used approach is first to decouple the MIMO process into two SISO processes with a pre-compensator and then to design SISO controllers for each decoupled subsystem (see, e.g., [2], [3], [4] [5]). To guarantee offset free tracking, integral control is recommended for each single loop. Although decoupling a multi-loop integral controller is easy to be implemented, it is not an optimal approach for the control of multivariable processes and tends to degrade their stability. In this paper model predictive control (MPC) is proposed in the design and implementation for the coupled drive system. MPC has been used in industry for more than 30 years, and has become an industry standard due to its intrinsic capability for dealing with constraints associated in multivariable systems. The main idea of MPC is to choose the control action by repeatedly solving on-line an optimization problem. This is aimed at minimizing a performance criterion over a future horizon, possibly subject to constraints on the manipulated inputs and outputs, where the future behavior is computed according to a model of the plant (http://www.pacontrol.com/MPC.html). As an optimal control approach, MPC has more advantages than classical multi-loop PID controller. For example, by selecting a proper weighting function, it has the potential of saving fuel of mobile vehicles.

In the literature, coupled drive systems are often treated as linear multivariable system. However, coupled drives in practice are quite often subject to nonlinear characteristics. In this laboratorial study, a linear MPC controller is designed for a linearized model around some selected operating point. The designed MPC controller is thus only valid in a certain operation range. When the operating point changes, the closedloop system performance may deteriorate. From our observation, for the control of coupled drives, the most difficult part for students' design is the handling of nonlinearityrelated issues in the system, such as, control input saturation, validation of operating point range, and model variation under different operational conditions. Students need assistance in the control design when some reference input values are chosen beyond the normal operating range. To theoretically explain all nonlinear effects involved, it may require more time allocation and effort with material that may be quite abstract and difficult at the undergraduate level. For example, in order to explain the nonlinear steady state conditions for multi-loop integral control 
[6] [7], some nonlinear control knowledge is needed beyond the knowledge of undergraduate students.

By using MPC control of the typical nonlinear multivariable system, this paper attempts to help students to understand fundamental concepts of abstract multivariable control for nonlinear processes. It presents an experiment-based approach to assist the learning of the advanced control theory, to show the decoupling capability of multivariable optimal control, and to facilitate students in designing their own multi-loop controllers.

This paper is organized as follows. Section II will briefly introduce the coupled drive system and followed by the modeling of the system. Section III presents the basic knowledge of MPC with an implementation guide. All the experimental results and discussion are provided in Section IV. A conclusion is drawn in Section V.

\section{MODELING OF THE COUPLED DRIVES SYSTEM}

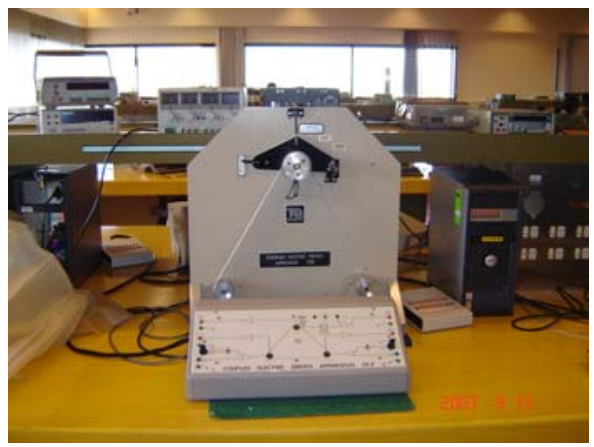

Fig. 1. Coupled Drives System

Figure 1 shows a coupled drive system. The parameters of this system are listed as follows:

- $\theta_{1,2}$ Motor $_{1,2}$ angular position (rad)

- $\theta_{3}$ Jockey pulley angular position (rad)

- $I_{1,2}$ Motor $_{1,2}$ inertia $\left(\mathrm{kg} \mathrm{m}^{2}\right.$ )

- $I_{3}$ Jockey pulley inertia $\left(\mathrm{kg} \mathrm{m}^{2}\right)$

- $m$ Jockey pulley mass ( $\mathrm{kg}$ )

- $k$ Belt stiffness $\left(\mathrm{Nm}^{-1}\right)$

- $k_{0}$ Jockey spring stiffness $\left(\mathrm{N} m^{-1}\right)$

- $b_{1,2}$ Motor friction $\left(\mathrm{kg} \mathrm{N} s^{-1}\right)$

- $b_{3}$ Pulley friction (angular $\mathrm{Nm}^{-1}$ )

- $b_{m}$ Pulley friction (translate $\mathrm{N}^{-1}$ )

The standard system has two drive motors which operate in cooperation to control simultaneously the tension and the speed of a continuous flexible belt and that goes round pulleys on the drive motor shafts with a jockey pulley. The jockey pulley is mounted on a swinging arm that is supported by a spring. In the manufacture of textiles, paper, wire, and plastic films, regulation of the tension and speed outputs of coupled drive systems are often needed.

From the existing literature, the dynamics of the system can be derived by using the Lagrange equations, and the initial values of the parameters of the model can be approximately estimated by using step and impulse responses.
The system's Lagrangian is written as $L=T-V$, where the kinetic energy is

$$
T=\frac{1}{2} m \dot{x}^{2}+\frac{1}{2} I_{1} \dot{\theta}_{1}^{2}+\frac{1}{2} I_{2} \dot{\theta}_{2}^{2},
$$

and the potential energy of the system is

$$
\begin{aligned}
& V=\frac{1}{2} k\left[r\left(\theta_{1}-\theta_{2}\right)\right]^{2}+\frac{1}{2} k\left[r\left(\theta_{1}-\theta_{3}\right)-x \cos (\alpha)\right]^{2} \\
& \quad+\frac{1}{2} k\left[r\left(\theta_{3}-\theta_{2}\right)-x \cos (\alpha)\right]^{2}+\frac{1}{2} k_{0} x^{2}
\end{aligned}
$$

Based on Lagrange equations, system dynamics can be derived as (see www.control-systems-principles.co.uk):

$$
M \ddot{z}+B \dot{z}+k z=U,
$$

where

$$
\begin{aligned}
& z=\left(\begin{array}{lll}
\theta_{1} & \theta_{2} & x
\end{array}\right)^{T}, U=\left(\begin{array}{lll}
u_{1} & u_{2} & 0
\end{array}\right)^{T} . \\
& M=\left(\begin{array}{ccc}
I_{1} & 0 & 0 \\
0 & I_{2} & 0 \\
0 & 0 & m
\end{array}\right), B=\left(\begin{array}{ccc}
b_{1} & 0 & 0 \\
0 & b_{2} & 0 \\
0 & 0 & b_{0}
\end{array}\right), \\
& \text { and } K=\left(\begin{array}{ccc}
\frac{3}{2} k r^{2} & -\frac{3}{2} k r^{2} & -\frac{\sqrt{3}}{2} k r^{2} \\
-\frac{3}{2} k r^{2} & \frac{3}{2} k r^{2} & \frac{\sqrt{3}}{2} k r^{2} \\
-\frac{\sqrt{3}}{2} k r^{2} & \frac{\sqrt{3}}{2} k r^{2} & k_{0}+\frac{3}{2} k
\end{array}\right) .
\end{aligned}
$$

The transfer function of the system can be obtained as:

$$
\left(\begin{array}{l}
w \\
x
\end{array}\right)=P(s)\left(\begin{array}{l}
u_{1} \\
u_{2}
\end{array}\right) .
$$

Where

$$
\begin{gathered}
P(s)=\left(\begin{array}{cc}
G_{w} & G_{w} \\
-G_{x} & G_{x}
\end{array}\right), \\
G_{w}=\frac{1}{2(I s+b)},
\end{gathered}
$$

and

$$
G_{x}=\frac{\sqrt{3} k r}{\left(I s^{2}+3 k r^{2}\right)\left(2 m s^{2}+2 k_{0}+3 k\right)-3 k^{2} r^{2}} .
$$

The control approach commonly recommended to the students for the control of the coupled drive system is multi-loop integral control. The main strategy for this control scheme is to transfer the $2 \mathrm{I} 2 \mathrm{O}$ system to two scalar systems by decoupling the control of Tension and Jockey speed. See Figure 2, a precompensator $K(s)$ is constructed to decouple the system:

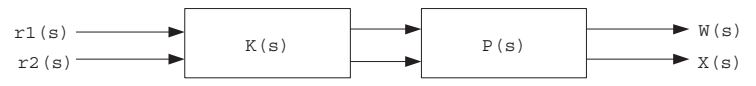

Fig. 2. Decoupling compensator.

Where

$$
K(s)=\left(\begin{array}{cc}
1 & -1 \\
1 & 1
\end{array}\right)
$$

Then,

$$
\left(\begin{array}{c}
w \\
x
\end{array}\right)=\left(\begin{array}{cc}
G_{w} & 0 \\
0 & G_{x}
\end{array}\right) K(s)\left(\begin{array}{l}
u_{1} \\
u_{2}
\end{array}\right) .
$$

The parameters can be approximately identified by using step and/or impulse response analysis. 


\section{Model Predictive Control}

Consider a nonlinear state space model of the form:

$$
\left\{\begin{array}{l}
\dot{x}(k)=f(x(k), u(k)), \\
y(k)=h(x(k), u(k)),
\end{array}\right.
$$

where $f(\cdot, \cdot)$ is Lipschitz continuous functions, twice differentiable, originated by differential equations representing system dynamics, and have the equilibrium points at the origin $f(0,0)=0$.

The control and state inequality constraints are deduced from their physical limits of the form $u(k) \in \mathcal{U}, x(k) \in \mathcal{X}$, where $\mathcal{U}$ is a convex, compact subset of $\mathcal{R}^{m}$ and $\mathcal{X}$ a convex, closed subset of $\mathcal{R}^{n}$, each set containing the origin in its interior. The control objective is to steer the state to the equilibrium point.

For the MPC problem, an online optimization problem is formed and solved at every time step using the initial values of state and control vectors $x(k-1)$ and $u(k-1)$. The objective function is defined by [8]

$J(x, u \mid \mathrm{x}(\mathrm{k}-1), \mathrm{u}(\mathrm{k}-1))=\sum_{i=k}^{k+N} \ell(x(i), u(i))+F(x(k+N))$.

The terminal time $k+N$ is referred to as a receding horizon or rolling horizon. Function $F(x(k-1))$ is the terminal cost function. A terminal constraint set of the form $x(k+N) \in$ $X_{f} \subset \mathcal{X}$ can be imposed for guaranteeing stability.

Once the constrained optimization problem of minimizing $J(x, u \mid \mathrm{x}(\mathrm{k}-1), \mathrm{u}(\mathrm{k}-1))$ subject to the equality constraints of the state space model and the inequality constraints of control and state vector, as well as terminal constraints is solved, the optimizing control sequence $\{u(k), u(k+1), \ldots, u(k+N)\}$ is yielded. The first control vector $u(k)$ in this sequence is applied to the system (at time $k$ ). As $N$ is finite, the minimum of $J(x, u \mid \mathrm{x}(\mathrm{k}-1), \mathrm{u}(\mathrm{k}-1))$ exists if $f(\cdot), \ell($.$) and F(\cdot)$ are continuous, $\mathcal{U}$ compact, and $\mathcal{X}$ and $\mathcal{X}_{f}$ closed.

For LTI systems, quadratic objective functions w.r.t. state, control and control increment vectors are normally considered. Predictive horizon for plant output vectors and control horizon for control input vectors are also normally selected when internal delays are taken into considerations. Due to linearity, different set-points are allowable, and the variables will take their non-zero steady states (or set-point) as references in the objective function. The objective function has a quadratic form of

$$
\begin{aligned}
& \quad J(x, u \mid \mathrm{x}(\mathrm{k}-1), \mathrm{u}(\mathrm{k}-1))= \\
& \quad \sum_{i=0}^{N_{y}}[y(k+i)-r]^{T} W_{y}[y(k+i)-r] \\
& +\sum_{i=0}^{N u}\left[u(k+i)-u_{s s}\right]^{T} W_{u}\left[u(k+i)-u_{s s}\right] \\
& +\sum_{i=0}^{N u} \Delta u(k+i)^{T} W_{\Delta u} \Delta u(k+i),
\end{aligned}
$$

where $W *$ are diagonal weighting matrices. The predictive (output) horizon is $N_{y}$, the control horizon is $N_{u}$.

With linear systems, it is more convenient to substitute the equality constraint of state space model directly into the objective function to result in the problem of quadratic programming for the minimizing sequence vector of input and/or input increment elements. This is the standardized MPC formulation for LTI systems that is available in Matlab MPC toolbox. The solution can be found using the mpcmove function in Matlab.

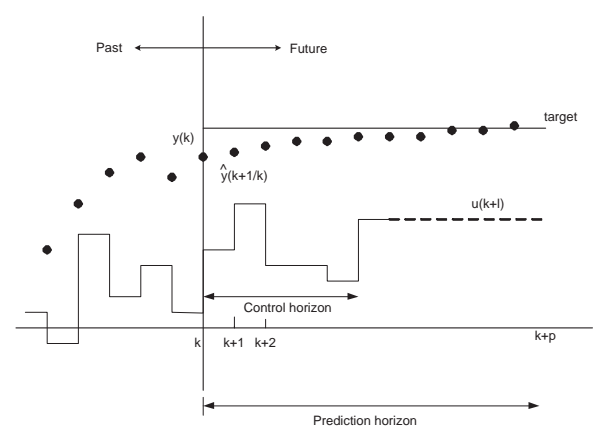

Fig. 3. MPC control

In our demonstration, the model predictive controller predicts and optimizes the future behaviour based on a dynamic model of the process. At each control interval, the MPC algorithm calculates an open loop sequence of the manipulated variables in such a way to optimize the future behaviour of the plant. The first value in this optimal sequence is injected into the plant. Figure 3 shows the state of a MPC system that has been operating for many sampling instants. Integer $k$ represents the current instant. The latest measured output, $y_{k}$, and previous measurements, $y_{k-1}, y_{k-2}, \ldots$, are known.

To calculate its next move $u_{k}$, the controller operates in two phases:

1. Estimation and prediction: In order to make an intelligent move, the controller needs to know the current state and any internal variables that influence the future trend. To accomplish estimation and prediction, the controller uses all past and current measurements and the models.

2. Optimization: Values of setpoints, measured disturbances, and constraints are specified over a finite horizon of future sampling instants, $k+1, k+2, \cdots, k+p$, where $p$ is the prediction horizon. The controller computes $m$ moves $u_{k}$, $u_{k+1}, \ldots u_{k+m-1}$, where $m$ is the control horizon. The moves are the solution of a constrained optimization problem:

$\min _{\Delta u_{k} \cdots \Delta u_{k+m-1}}\left(\sum_{l=1}^{p}\left\|\hat{y}_{k+l / k}-r_{k+l}\right\|_{\Gamma_{l}^{y}}^{2}+\sum_{l=1}^{m}\left\|\Delta u_{k+l-1}\right\|_{\Gamma_{l}^{u}}^{2}\right)$,

where,

- $\hat{y}_{k+l / k}$ is the predicted values of $y$ at time $k+l$ based on information available at time $k$.

- $p$ is prediction horizon which sets the number of control intervals over which the controller predicts its outputs when computing controller moves.

- $m$ is control horizon which sets the number of moves computed. It must not exceed the prediction horizon. If less than the prediction horizon, the final computed move fills the remainder of the prediction horizon. 
- $\Delta u_{k}=u_{k}-u_{k-1}$.

- $\|x\|_{\Gamma}^{2}=x^{T} \Gamma x$.

- $\Gamma_{l}^{y}$ and $\Gamma_{l}^{u}$ are weighting matrices for predicted errors and control moves $\left(\Gamma_{l}^{y}>0\right.$ and $\left.\Gamma_{l}^{u} \geq 0\right)$.

For implementation in Labview and Matlab environment, the MPC object is formulated with the $m p c($ ) function with an initial state created for the MPC object:

$M P C \_O b j \_C D S=m p c\left(M o d e l \_C D S, T s, P y, P c\right.$,

Weights, InputSpecs, OutputSpecs);

$x m p c=$ mpcstate $\left(M P C \_O b j \_C D S\right)$;

where the predictive horizon and control horizon are declared in $P y$ and $P c$.

With the output and reference vectors declared as $y$ and $r e f$ respectively, the manipulated input vector $u$ is obtained from the following iteration using a for loop:

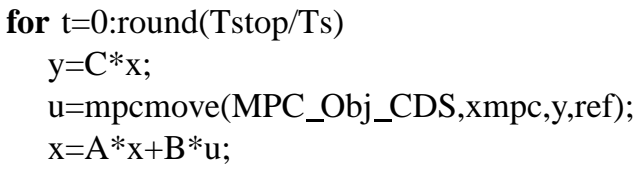

where Tstop is the user-defined maximum time scale.

\section{EXPERIMENTAL RESULTS AND DISCUSSIONS}

The proposed model predictive control algorithm was implemented by using Labview and Matlab Model Predictive Control Toolbox. The model structure has been given in Section 2 with given numerical values of parameters given that can be found at www.control-systems-principles.co.uk. Here the parameter set is used as initial values for the model coefficients, and trial-and-error will be applied to fine tune these coefficients around a particular operating point. We will show later the control performance in dependence on the operating point due to the nonlinear characteristics of the system. The best model around a particularly-selected operating point $\left(r_{1}=3 V\right.$ and $\left.r_{2}=3 V\right)$ is presented as follows:

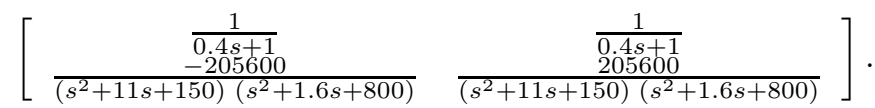

The control results corresponding to this model are given in Figures 4 and 5 under different control input rate constraints. We also generated an external disturbance around $t=50$ seconds as shown in Figure 4. The system can still perform fast tracking the reference input under such a big disturbance. It should be mentioned that the decoupling compensator has not been constructed explicitly in the MPC controller. This is to demonstrate that the Model Predictive Controller itself can handle (i) the coupling effect based on model predicted dynamics, and furthermore, (ii) the saturation effect by simply imposing constraints on the input voltages.

We need to emphasize that the reference inputs for angular velocity and belt tension are $3 \mathrm{~V}$ and $3 \mathrm{~V} \quad\left(r_{1}=3 \mathrm{~V}\right.$ and $\left.r_{2}=3 V\right)$ respectively. When the reference input is changed, the operating point will change. The control performance may degrade and the system even becomes unstable. Figures 6, 7, and 8 show the performance under different reference values. It
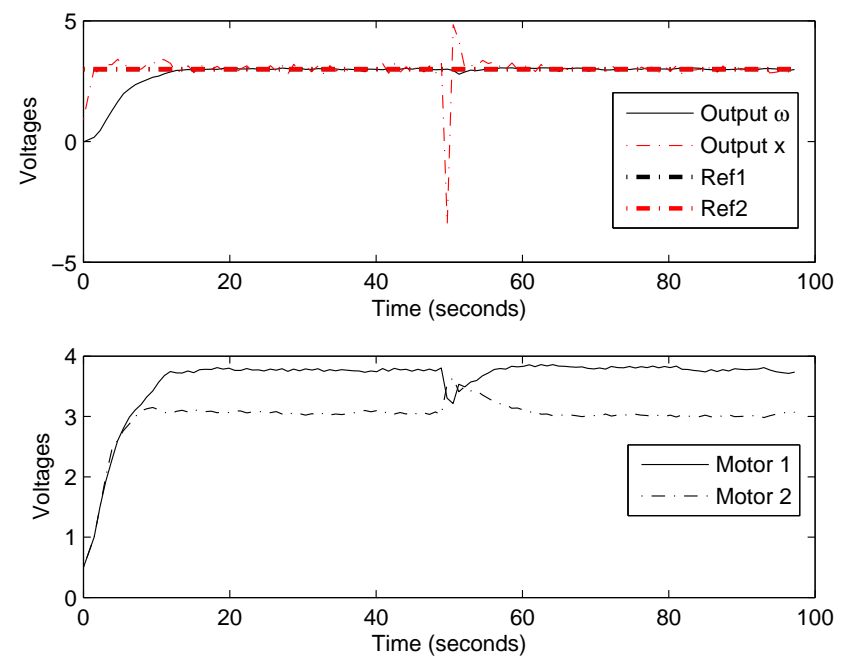

Fig. 4. Best performance with rate constraint $[-0.5 \mathrm{~V} /$ Sample, $-0.5 \mathrm{~V} /$ Sample $]$ and reference inputs as $r_{1}=3 \mathrm{~V}$, and $r_{2}=3 \mathrm{~V}$.
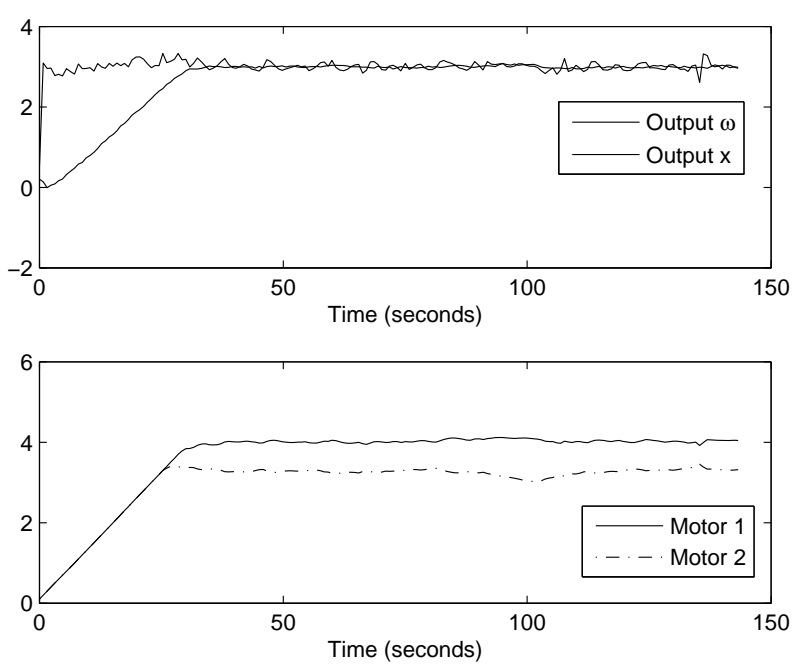

Fig. 5. Best performance with rate constraint $[-0.1 \mathrm{~V} /$ Sample, $-0.1 \mathrm{~V} /$ Sample $]$ and reference inputs as $r_{1}=3 \mathrm{~V}$, and $r_{2}=3 \mathrm{~V}$.

can be seen that the best control performance achieved is under the reference input $r_{1}=1-3 V$ and $r_{2}=2-3 V$. For small reference inputs (e.g. $r_{1}=0 \mathrm{~V}$ and $r_{2}=0 \mathrm{~V}$ ), the closed-loop system is unstable. For some reference inputs (e.g. $r_{1}=3 \mathrm{~V}$ and $r_{2}=4 \mathrm{~V}$ ), the transient performance of the closed-loop system degrades. This indicates that the identified model is only valid in a certain operating range, and the coupled drive system certainly exhibits nonlinear behaviors. This would be a practical example to show that real systems, strictly speaking, are nonlinear in nature.

The MPC is actually an integral control scheme. For a multivariable nonlinear system, it is not always possible to build an integral controller in some operating range of the system. If it 

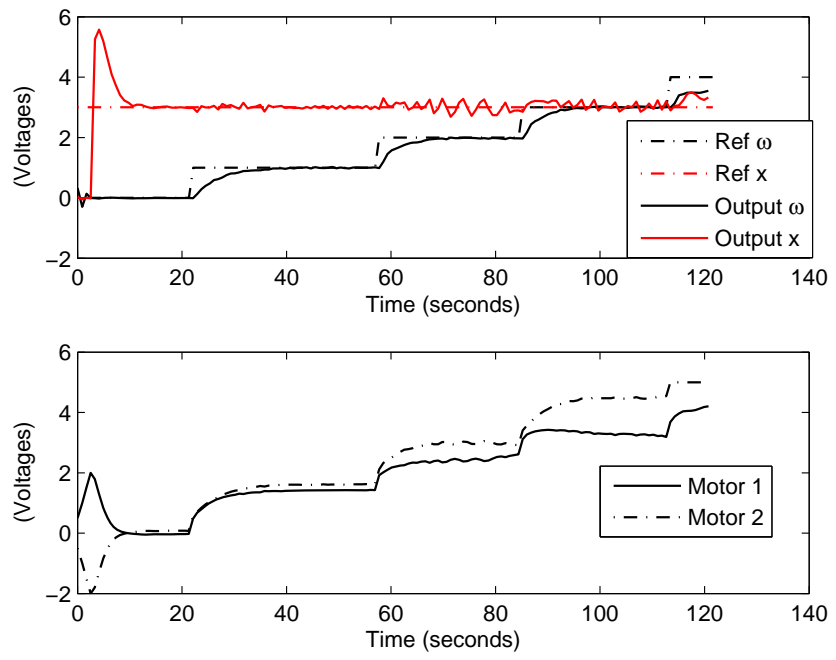

Fig. 6. Tracking performance with rate constraints $[-0.5 \mathrm{~V} /$ Sample, $-0.5 \mathrm{~V} /$ Sample $]$ and reference inputs as $r_{1}=0,1,2,3,4,5 V$, and $r_{2}=3 \mathrm{~V}$.
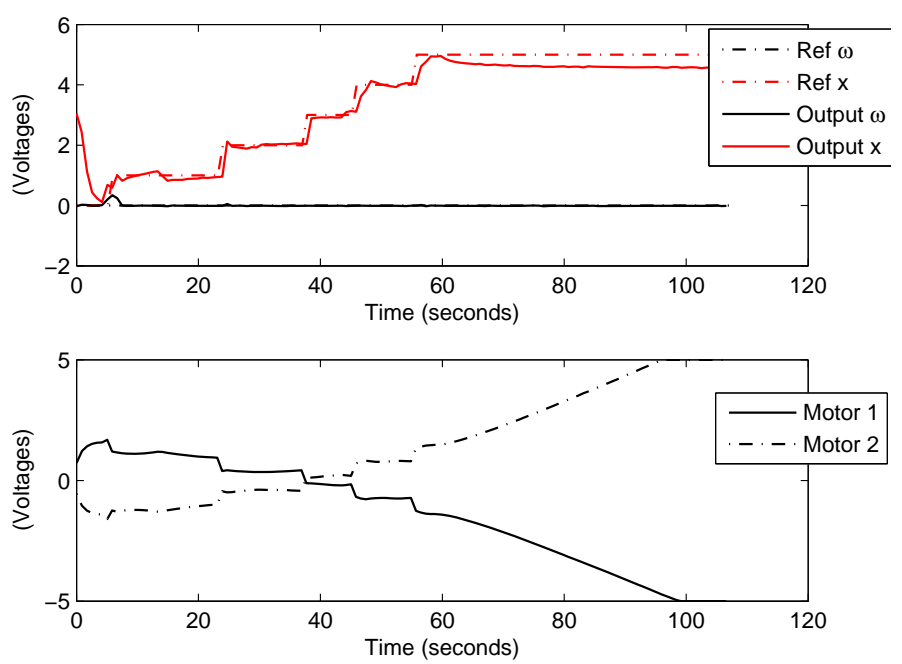

Fig. 7. Tracking performance with rate constraints $[-0.5 \mathrm{~V} /$ Sample, $-0.5 \mathrm{~V} /$ Sample $]$ and reference inputs as $r_{1}=0 \mathrm{~V}$, and $r_{2}=0,1,2,3,4,5 \mathrm{~V}$.

is impossible to track a given reference signal by a centralized integral controller, so is it by a decentralized integral controller. Therefore, it may be helpful for the instructors to identify the range of reference signals to assist students with their design of a multi-loop integral controller for model predictive control of coupled drive systems.

Control saturation is another practical issue in engineering practice. For a control input, the commonly-encountered constraint is saturation as all the input variables have physical limitations. For the coupled drive system in our laboratory, the two voltage inputs of the motors are limited from $-5 \mathrm{~V}$ to $+5 \mathrm{~V}$. In order to ensure the safety of the system, at the initial stage, we can select a rate constraint
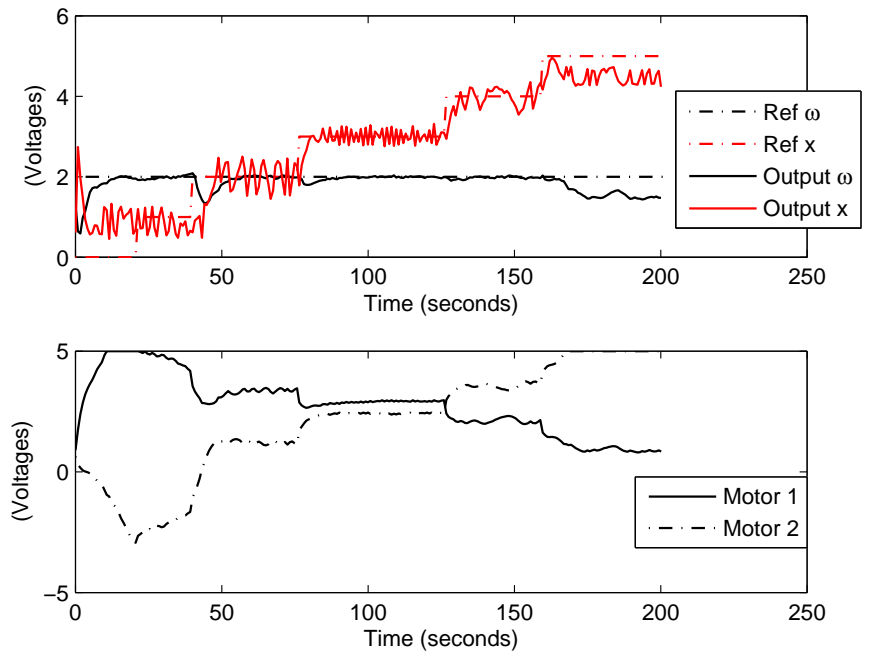

Fig. 8. Tracking performance with rate constraints $[-0.5 \mathrm{~V} /$ Sample, $-0.5 \mathrm{~V} /$ Sample $]$ and reference inputs as $r_{1}=2 \mathrm{~V}$, and $r_{2}=0,1,2,3,4,5 \mathrm{~V}$.
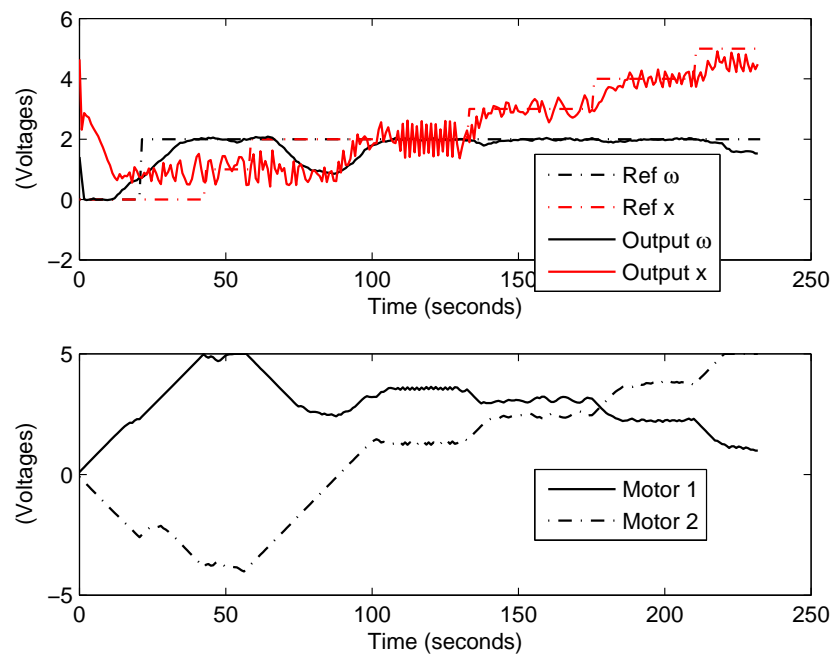

Fig. 9. Tracking performance with rate constraints $[-0.1 \mathrm{~V} /$ Sample, $-0.1 \mathrm{~V} /$ Sample $]$ and reference inputs as $r_{1}=2 \mathrm{~V}$, and $r_{2}=0,1,2,3,4,5 \mathrm{~V}$.

for the input voltages as well. In this study, the rate constraint is selected as $[-0.1 \mathrm{~V} /$ Sample, $-0.1 \mathrm{~V} /$ Sample $]$ and $[-0.5 \mathrm{~V} /$ Sample, $-0.5 \mathrm{~V} / \mathrm{Sample}]$. From experimental results (in comparison of Figures 9 and 10 with Figure 8), we can see that $[-0.5 \mathrm{~V} /$ Sample, $-0.5 \mathrm{~V} /$ Sample $]$ is better. With this rate constraint, the control performance is not affected and the safety of the system can also be improved as well.

We also investigated another important issue for discrete time control systems, i.e. controller update rate. In real-world applications, students may confuse between the sampling rate of the sensor and the controller update rate in the feedback system. In this example, we try to use experiments to show the importance of selecting a correct controller update rate. 

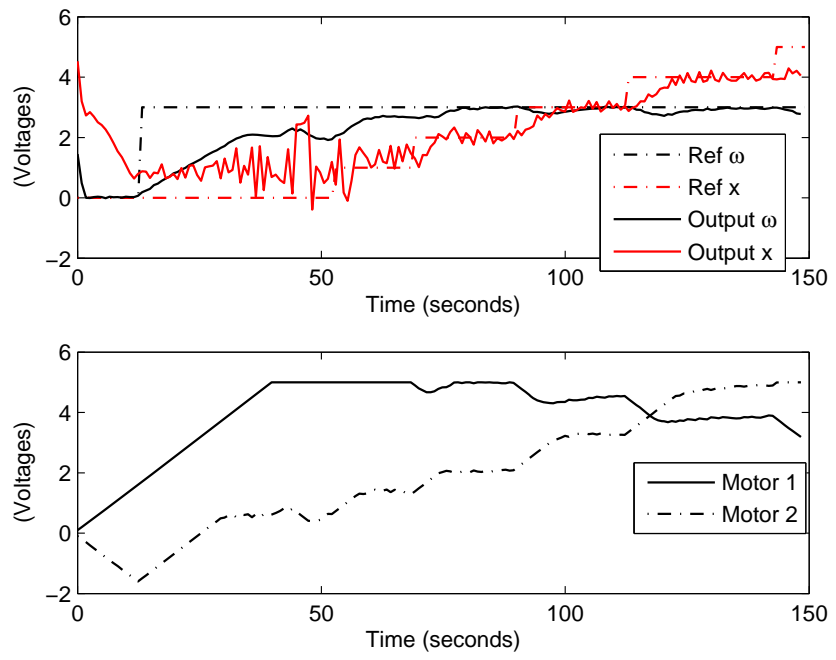

Fig. 10. Tracking performance with rate constraints $[-0.1 \mathrm{~V} /$ Sample, $-0.1 \mathrm{~V} /$ Sample $]$ and reference inputs as $r_{1}=3 \mathrm{~V}$, and $r_{2}=0,1,2,3,4,5 \mathrm{~V}$.
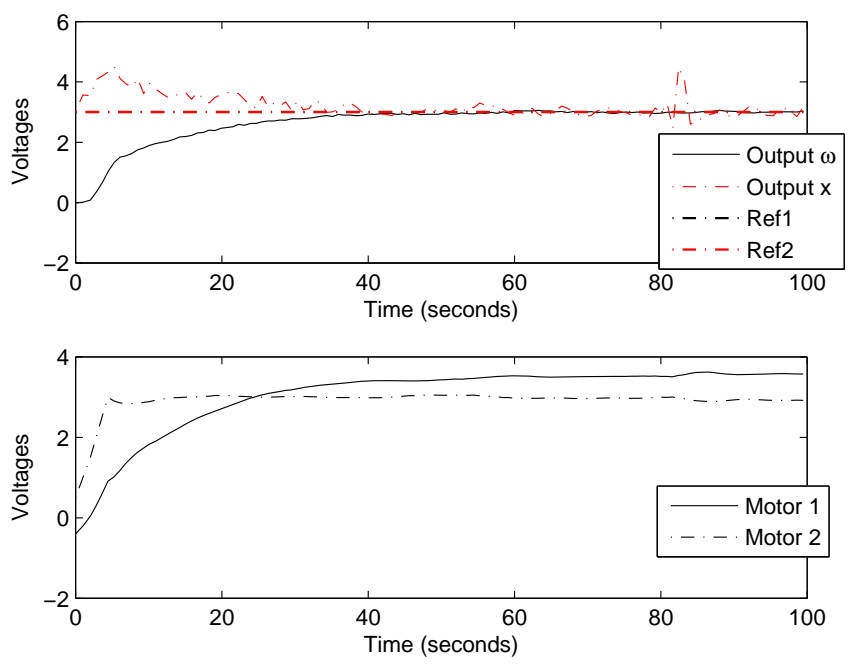

Fig. 11. Tracking performance with rate constraints $[-0.1 \mathrm{~V} /$ Sample, $-0.1 \mathrm{~V} /$ Sample $]$ and reference inputs as $r_{1}=3 \mathrm{~V}$, and $r_{2}=0,1,2,3,4,5 \mathrm{~V}$; controller update rate $f_{c}=5 \mathrm{~Hz}$.

For this MPC system, the controller update rate is selected as $f_{c}=1.25 \mathrm{~Hz}$. The sampling rate of angular velocity sensor and belt tension sensor are $f_{s}=5 \mathrm{~Hz}$. In Figure 11, we show the bad effect when we select $f_{c}=f_{s}=5 \mathrm{~Hz}$ for the controller update rate, as quite often by mistake. Figure 11 shows that the tracking becomes slower even under well-chosen references $r_{1}=3 \mathrm{~V}$ and $r_{2}=3 \mathrm{~V}$. However, if we select a slower controller update rate (e.g. $f_{c}=0.5 \mathrm{~Hz}$ ), the closed loop system may become unstable. This is a good example to show the importance of controller update rate selection.

\section{CONCLUSION}

This paper has presented some laboratory demonstrations for the design and implementation of a model predictive controller for a multivariable process that is typically-encountered in industrial manufacturing and automation, the coupled drive system. The effort is motivated by enhancement of undergraduate control education quality. From the general formulation of model predictive control for nonlinear systems, we showed the controller development including some implementation instruction for the coupled drive apparatus. Based on experimental results, the effectiveness of this optimal control approach is verified for a multi-input multi-output system in general. Furthermore, illustrative explanations are included for several identified issues in relevance to automation and control practice and education. It is expected that this work can serve to inspire interests in control engineering and to facilitate, motivate the teaching and learning of control-related subjects.

\section{REFERENCES}

[1] J. Kocijan, J. O'Reilly, and W. Leithead, "An integrated undergraduate teaching laboratory approach to multivariable control," IEEE Trans. Educ., vol. 40, no. 4, pp. 266-272, 1997.

[2] V. Nguyen, H. Nguyen, and Q. Ha, "Sliding mode neural controller for nonlinear systems with higher-order and uncertainties," IEEE Conference on Robotics, Automation and Mechatronics, pp. 1026-1031, 2004.

[3] M. Kubalcik and V. Bobal, "Application of decoupling controllers to coupled drives process," In: Proc. of the 5th Asian Control Conference, pp. 1446-1451, 2004.

[4] R. Paz, B. Garcia, and J. Torres, "Fuzzy-genetic controller for a coupled drives system," IEEE International Symposium on Industrial Electronics, Puebla, Mexico, pp. 741-746, 2004.

[5] J. Pieper and B. Surgenor, "Discrete sliding control of a coupleddrives apparatus with optimal sliding surface and switching gain," IEE PROCEEDINGS-D, vol. 140, no. 2, pp. 70-78, 1993.

[6] P. Campo and M. Morari, "Achievable closed-loop properties of systems under decentralized control: conditions involving the steady-state gain," IEEE Trans. Automat. Con., vol. 39, no. 5, pp. 932-942, 1994.

[7] S. Su, J. Bao, and P. Lee, "Analysis of decentralized integral controllability for nonlinear systems," Computers and Chemical Engineering, vol. 28, no. 9, pp. 1781-1787, 2004.

[8] J. Maciejowski, Predictive Control with Constraint. Harlow: Pearson Education, 2001. 